Molecular Science, Barts and The London School of Medicine \& Dentistry, London, UK; ${ }^{2}$ Division of Infection \& Immunity, UCL, London, UK; ${ }^{3}$ Department of Virology, Barts and The London NHS Trust, London, UK

Introduction Stopping-rules now exist for Pegylated Interferon- $\alpha$ (PEG-IFN $\alpha$ ) treated Chronic Hepatitis B (CHB) patients. Despite the utility of such strategies, the immunological mechanisms that drive HBV DNA and HBsAg decline remain poorly understood. Recent data have identified changes in a subset of NK cells in $\mathrm{HBeAg}$ negative disease, which may determine treatment response. However, HBeAg positive disease responds more favourably to PEGIFN $\alpha$; here we report on a longitudinal analysis of changes in the immune profile in this cohort, to define the effects of PEG-IFN $\alpha$ on innate immunity.

Methods PBMCs from a cohort of $17 \mathrm{HBeAg}$ positive patients followed longitudinally at 3 monthly intervals pre, during and post PEG-IFN $\alpha$ therapy were utilised. Phenotypic analysis of NK cells was performed by multicolour flow cytometry. Changes in the immune responses were correlated with simultaneous measurements of ALT, HBV DNA and quantitative HBsAg levels (Abbott ARCHITECT).

Results PEG-IFN $\alpha$ increased CD56 ${ }^{\text {bright }}$ NK cells by fourfold (mean fold change; MFC 3.7, $\mathrm{p}=0.0001$ ). This was parallelled by the activation and proliferation of this subset, as marked by HLA-DR and Ki67 expression respectively (MFC 1.5 and 2.3, $p=0.009$ and $\mathrm{p}=0.0001$ respectively). This increase was more marked at 48 weeks treatment, correlating with a nadir of HBV DNA and HBsAg. The activating (NKG2C and NKp30) and inhibitory (NKG2A) receptors were also analysed in this population. A twofold increase in NKp30 expression (MFC 2.27, $\mathrm{p}=0.04$ ) was seen which was maximal at 48 weeks, while no significant change was noted for NKG2A and NKG2C. There was a twofold up-regulation of TRAIL expression on CD56 ${ }^{\text {bright }} \mathrm{NK}$ cells, which temporally correlated with ALT levels, (MFC 1.8, $\mathrm{p}=0.0001$ ), this effect was most dramatic at 24 weeks of therapy and sustained to 48 weeks.

Conclusion PEG-IFN $\alpha$ therapy in this cohort enhances and activates CD56 $6^{\text {bright }}$ NK cells. Similarly, TRAIL and NKp30 expression is augmented and sustained throughout treatment and all these effects are maximal at 48 weeks. The restorative innate immune changes begin early and increase throughout therapy in all patients. Thus, 48 weeks therapy may provide the optimal immunological conditions to introduce an oral-antiviral to achieve disease control in PEG-IFN $\alpha$ non-responders.

Competing interests None declared.

\section{PM0-178 THE SIGNIFICANCE OF VIRAL AND SEROLOGICAL MARKERS IN PREDICTING LIVER DISEASE SEVERITY IN E-AG NEGATIVE HEPATITIS B VIRUS INFECTION}

\section{doi:10.1136/gutjnl-2012-302514b.178}

V K Audimoolam,* I Carey, M Bruce, M Horner, S Abid, K Agarwal, P Harrison. Institute of Liver studies, King's College Hospital, London, UK

Introduction In hepatitis B virus (HBV) infection, seroconversion to $\mathrm{HBeAg}$ negative/eAb positive accompanied by low serum HBVDNA (persistently $\leq 2000 \mathrm{IU} / \mathrm{ml}$ ) and low quantitative $\mathrm{HBsAg}$ (qHBsAg) signifies transition to an inactive carrier (IC) state. However, in patients with raised serum HBV DNA differentiating those with inactive disease (ID) from eAg negative chronic HBV (eAg-CHB) currently relies on liver biopsy. This study investigated whether serological (qHBsAg) and virological markers (serum HBV DNA) can predict disease severity in patients with eAg negative $\mathrm{HBV}$ across a range of HBV genotypes.

Methods Liver biopsy was performed in 364 consecutive eAg negative patients (median age 38 years, 212 males) who had HBV DNA
$>1000 \mathrm{IU} / \mathrm{ml}$ on at least two clinic visits over 6-18 months. ALT [IU/1], qHBsAg [Abbott ARCHTECT $®$ ], HBV genotype [direct sequencing] and HBV DNA [real-time TaqMan PCR] were evaluated at the time of liver biopsy.

Results Based on the liver histology findings, 217 had ID (an Ishak fibrosis score of F0-1) and 147 had eAg-CHB ( $\geq \mathrm{F} 2)$. HBV genotype-E predominated $(50 \%)$ followed by $\mathrm{D}(16 \%), \mathrm{A}(15 \%), \mathrm{B}(10 \%)$ and $\mathrm{C}$ (9\%). Overall qHBsAg levels were higher in ID than eAg-CHB patients (median 3.84 vs $3.7 \log _{10} \mathrm{IU} / \mathrm{ml} ; \mathrm{p}=0.02$ ). Assessment by individual genotype demonstrated that $\mathrm{qHBsAg}$ levels remained higher in ID than eAg-CHB in genotypes A and E (4.01 vs 3.73 and 3.95 vs $3.8 \log _{10} \mathrm{IU} / \mathrm{ml}$; both $\left.\mathrm{p}<0.05\right)$. However, in genotype $\mathrm{B}$ qHBsAg levels correlated with the severity of fibrosis [2.81 in F0-1 vs 3.34 in $\mathrm{F} \geq 2 ; \mathrm{p}<0.01]$. The $\mathrm{qHBsAg}$ levels were similar in ID and eAg-CHB in genotypes $C$ and D. HBV genotype had no impact on the severity of liver fibrosis $(p=0.16)$. Patients with eAg-CHB compared to those with ID had raised ALT [ $81 \%$ vs $65 \%$; $<<0.01$ ], higher HBV DNA (3.99 vs $3.6 \log _{10} \mathrm{IU} / \mathrm{ml} ; \mathrm{p}<0.01$ ), older age (39 vs 36 years; $\mathrm{p}<0.01)$ and more were males $(68 \%$ vs $51 \%$; $\mathrm{p}<0.01)$.

Conclusion In eAg negative patients with HBV DNA >1000 IU/ml, the relationship between qHBsAg levels and liver fibrosis was genotype specific. Even allowing for HBV genotype, the absolute qHBsAg level was a poor discriminator of clinically significant liver fibrosis.

Competing interests V Audimoolam: None declared, I Carey Grant/Research Support from: Gilead, M Bruce: None declared, M Horner: None declared, S Abid None declared, K Agarwal: None declared, P Harrison: None declared.

\section{PM0-179 ROLE OF ANTIVIRALS IN ACUTE HEPATITIS B INFECTION; A 5-YEAR EXPERIENCE AT A LIVER TRANSPLANT CENTRE}

doi:10.1136/gutjnl-2012-302514b.179

${ }^{1} \mathrm{~V}$ S Hegade, ${ }^{*} \mathrm{~N}$ Mohammed, ${ }^{1} \mathrm{M}$ Aldersley, ${ }^{2} \mathrm{~S}$ Riyaz. ${ }^{1}$ Liver Transplant Unit, St James Hospital, Leeds, UK; ${ }^{2}$ Gastroenterology and Hepatology Department, Barnsley Hospitals NHS Foundation Trust, Barnsley, UK

Introduction Acute hepatitis B in adults is successfully cleared in more than $95 \%$ of immunocompetent patients. A small proportion of patients develop fulminant hepatitis. Few controlled trials ${ }^{12}$ have evaluated the role of antivirals in patients with acute severe hepatitis B (AS-HBV). The aim of this study was to report our experience of AS-HBV management at a tertiary centre.

Methods We retrospectively identified all patients between August 2006 and August 2011, referred to our centre with acute HBV infection (diagnosis based on recent onset of jaundice, detection of serum $\mathrm{HBsAg}$ and IgM $\mathrm{HBcAb}$ ). 1-Year data following the diagnosis was collected using medical and electronic records. We identified all patients meeting at least one of the three criteria for $\mathrm{AS}^{-\mathrm{HBV}^{1}}$ that is, INR $\geq 1.6$, serum bilirubin $\geq 170 \mu \mathrm{mol} / 1$ and hepatic encephalopathy. Patients with other causes of acute liver injury such as alcohol and drugs were excluded.

Results 98 patients with acute HBV were identified during the study period. Of these, $64(65.3 \%)$ patients had milder episodes. Thirty-four patients (34.7\%; mean age $32,50 \%$ females) had evidence of AS-HBV. Out of these, 17 (50\%) patients had a bilirubin $\geq 170 \mu \mathrm{mol} / 1$ and $3(8.8 \%)$ patients had INR $\geq 1.6$ while $14(41 \%)$ patients had both. None of the AS-HBV patients had evidence of encephlopathy. All patients had normal ultrasound scans of the liver and negative non-invasive liver screen, at the time of diagnosis. Of the 34 patients with AS-HBV, 20 (59\%) patients received treatment with antiviral drugs, 55\% with newer agents (Entecavir or Tenofovir) and $45 \%$ with older agents (Lamivudine or Adefovir). None of the patients developed any side effects to antiviral drugs. The remaining 14 (41\%) patients with AS- HBV received supportive care 
only. There were no deaths or need for liver transplantation in either group.

Conclusion Acute hepatitis B usually runs a benign course but a proportion of patients can develop severe disease. Patients with acute severe hepatitis B may be safely treated with anti-viral drugs. Randomised controlled studies with newer antiviral agents are required to establish guidelines in treating patients with acute severe hepatitis B.

Competing interests None declared.

\section{REFERENCES}

1. Schmilovitz-Weiss $\mathbf{H}$, et al. Lamivudine treatment for acute severe hepatitis $\mathbf{B}$ : a pilot study. Liver Int 2004;24:547-51.

2. Kumar $\mathbf{M}$, et al. A randomized controlled trial of lamivudine to treat acute hepatitis $\mathbf{B}$. Hepatology 2007; 45:97-101

\section{PM0-180 RAISED SERUM IMMUNOGLOBULINS IN CHRONIC HEPATITIS C: INCIDENCE AND ASSOCIATION WITH GENOTYPE, LIVER FIBROSIS AND SUSTAINED VIRAL RESPONSE}

doi:10.1136/gutjnl-2012-302514b.180

I-U Haq, V S Hegade, ${ }^{*}$ K Forrester, P B Southern, S M Moreea. Department of Hepatology, Digestive Disease Centre, Bradford Teaching Hospitals Foundation NHS Trust, Bradford, UK

Introduction Serum Immunoglobulins (Igs) are commonly raised in Chronic Hepatitis C (HCV) but their clinical significance is not fully known. There is also little information on the normalisation of Igs post HCV treatment. We aimed to assess (1) the incidence of raised Igs in HCV patients, (2) the association between the most commonly raised Ig [Immunoglobulin (IgG)] and genotype [G] and liver fibrosis and (3) the association between normalisation of $\operatorname{IgG}$ in those achieving sustained viral response (SVR).

Methods Demographics, genotype, pre-and post-treatment Igs, Ishak liver fibrosis scores (F) and SVR of all patients undergoing treatment for HCV since 2006 was collected. Data from G2, G4, G6 and unknown genotype patients were not included in the analyses. Results 295 (n) patients were treated in the study period \{Genotype 1 [G1] 71, Genotype 3 [G3] 205, males 181 (mean age 45.4); females 114 (mean age 41.3$)\}$.

1. $217 / 295(73 \%)$ patients had raised pre-treatment Igs-either alone or in combination. Raised pre-treatment IgG, IgM and IgA were seen in $32 \%, 22 \%$ and $11 \%$ of G1 and in $56 \%, 16 \%$ and $8 \%$ of G3 patients respectively. A significant association between viral genotype and raised pre-treatment level was seen only with $\operatorname{IgG}(p=0.0009)$ and not with $\operatorname{IgA}(p=0.46)$ or $\operatorname{IgM}(p=0.20)$.

2. In G1, $43 \%$ of patients with advanced fibrosis $(F>4)$ had raised pre-treatment IgG compared to $29 \%$ of patients with $\mathrm{F} \leq 4$ [non-significant (NS) association, $\mathrm{p}=0.66$ ]. However in G3 advanced fibrosis $(\mathrm{F}>4)$ was significantly associated with raised pre-treatment IgG [33/41 (80\%) with $\mathrm{F}>4$ vs $70 / 130$ $(54 \%)$ with $\mathrm{F} \leq 4, \mathrm{p}=0.0031]$ suggesting that pre-treatment $\mathrm{IgG}$ can be a good predictor of advanced fibrosis in G3.

3. Overall SVR was achieved in 34\% in G1 and 65\% in G3. In those who achieved SVR, normalisation of raised IgG was seen more in G3 than in G1 [52\% vs $44 \%$, NS association, $\mathrm{p}=0.72]$.

Conclusion Our study confirms: (1) Presence of raised serum immunoglobulins, particularly that of IgG is common in both G1 and G3 patients. (2) Significant association between raised pretreatment IgG and advanced fibrosis is seen in G3 but not in G1. In G3, pre-treatment IgG level can be good predictor of advanced fibrosis. (3) Post-SVR normalisation of IgG is seen more in G3 than in G1.

Competing interests None declared.

\section{PM0-181 LONG TERM FOLLOW-UP OF CHRONIC HEPATITIS B (HBV) PATIENTS TREATED WITH PEGYLATED INTERFERON:} SINGLE CENTRE EXPERIENCE

doi:10.1136/gutjnl-2012-302514b.181

W Smethurst, * L Fenton, K Forrester, R Simpson, S Moreea. Bradford Teaching Hospitals Foundation Trust, Bradford, UK

Introduction Pegylated Interferon (PEG IFN) is NICE approved for the treatment of chronic hepatitis B (HBV) but it is now known that the response is genotype dependent. To analyse the long term outcome of chronic hepatitis B patients treated with Pegylated Interferon.

Methods Retrospective analysis of our hepatitis B database to identify HBV patients treated with PEG IFN. The following data were obtained from the patient's records and our electronic reporting systems: demographics, length of treatment, ALT and viral load (HBV PCR) at various points during and after treatment.

Results 13 patients ( 9 males, average age 36.8 yrs; 4 females, average age 34.7 yrs) were treated from April 2007 with a mean follow-up of 33 months (133 weeks). There were $8 \mathrm{eAg}+\mathrm{ve}$ and $5 \mathrm{eAg}-\mathrm{ve}$ patients. None were co-infected with the Delta virus. In the eAg +ve group, there were 3 genotype $\mathrm{D}$ (South Asians), 3 genotype C (2 Chinese and 1 South Asian), 1 genotype B (South Asian) and 1 unknown genotype (white Caucasian). All had a raised ALT and mild changes (Ishak fibrosis score $0-2$ ) on liver biopsy. 4/8 (50\%)-3 Genotype C, 1 Genotype D-achieved eAg seroconversion to eAb +ve and 1/8 (13\%)-unknown genotype-achieved sAg clearance at the end of treatment. ALT normalised only in those who seroconverted. HBV PCR was $<200 \mathrm{IU} / \mathrm{ml}$ in $5 / 8$ patients at week 24 and $4 / 8$ patients had undetectable PCR at the end of treatment at week 48 (lack of data for two patients, one failed treatment and one HBV PCR $42 \mathrm{IU} / \mathrm{ml}$ ). There were three cases of lamivudine resistance. Four patients relapsed within 1-year post PEG IFN (all genotype $\mathrm{D})$ and required treatment with tenofovir (+/- lamivudine). In the eAg - ve group, there were four genotype D patients (all South Asians, two co-infected with HCV genotype 3a) and 1 unknown genotype (Chinese). The three non co-infected patients showed good response at the end of treatment but all relapsed within 1 year and all needed further treatment with nucleoside analogues with good viral response. The co-infected patients achieved sustained viral response for HCV and maintained a low HBV viral load.

Conclusion This study confirms good outcomes for non-genotype $\mathrm{D}$ patients treated with PEG IFN. However, eAg -ve patients with genotype D treated with PEG IFN tend to relapse after treatment. The use of HBsAg quantification will help to tailor treatment in the future.

Competing interests None declared.

\section{PMO-182 HCV RESEARCH UK: A UK NATIONAL RESOURCE TO SUPPORT RESEARCH INTO HCV INFECTION}

doi:10.1136/gutjnl-2012-302514b.182

${ }^{1}$ W Irving, ${ }^{* 2} \mathrm{~J}$ McLauchlan, ${ }^{3} \mathrm{G}$ Foster, ${ }^{4} \mathrm{~J}$ Dillon, ${ }^{5} \mathrm{~S}$ Hutchinson, ${ }^{1} \mathrm{~B}$ Wilkes. ${ }^{1}$ University of Nottingham, Nottingham, UK; ${ }^{2} \mathrm{MRC} /$ University of Glasgow Centre for Virus Research, Glasgow, UK; ${ }^{3}$ University of London, London, UK; ${ }^{4}$ University of Dundee, Dundee, UK; ${ }^{5}$ Health Protection Scotland, Glasgow, UK

Introduction Background: Hepatitis $\mathrm{C}$ virus (HCV) infection has been identified by the MRC and Department of Health MRC as a 